\title{
Les entreprises marocaines et le développement durable: défis et enjeux. Illustration par le cas de l'OCP (site de Youssoufia)
}

\author{
Rachid Zammar \\ Professeur Habilité à la faculté des sciences de Rabat. Université \\ Mohammed V. Maroc \\ Noureddine Abdelbaki \\ Professeur Habilité à la faculté des sciences juridiques économiques et \\ sociales d'Agadir. \\ Université Ibn Zohr. Maroc
}

doi: 10.19044/esj.2016.v12n29p307 URL:http://dx.doi.org/10.19044/esj.2016.v12n29p307

\begin{abstract}
This article analyzes the challenges that the Moroccan companies namely Morocco's Office Cherifien of Phosphate (OCP) in the Youssoufia site have to address in order to move towards sustainable development. It examines the necessary strategies for the implementation of such an approach and highlights the issues necessary for such a vision. It aims to answer the following question: what is the impact of the projects introduced by the OCP on the development of the city of Youssoufia? The research is based on a qualitative study conducted with some group leaders and directors of public institutions in the city of Youssoufia through semi-structured interviews. The main results of the evaluation are as follows:

-at the environmental level: though the group has carried out several projects they do not help improve air quality nor reduce urban sprawl;

- at the social level: the group has conducted several civic actions that are limited in time and do not help those who really need help. However, funding for associations' activities remains reserved and unsustainable, many unfulfilled commitments as the schools that have no adequate water facilities.
\end{abstract}

Keywords: Sustainable development, OCP, social and environmental responsibility 


\section{Résumé}

Cet article tente d'analyser les défis que doivent relever les entreprises marocaines notamment le groupe OCP (notre étude de cas) dans son site de Youssoufia pour se diriger vers le développement durable, examiner les stratégies permettant la mise en place d'une telle démarche et mettre l'accent sur les enjeux de l'adhésion à une telle vision. Il a pour objet de répondre à la problématique suivante : quel est l'impact des projets initiés par l'OCP sur le développement de la ville de Youssoufia? Il s'est basé sur une étude qualitative menée auprès de quelques responsables du groupe et les directeurs des institutions publiques au sein de la ville via des entretiens semi-directifs.

Les principaux résultats de l'évaluation sont comme suit:

- au niveau environnemental, le groupe a réalisé plusieurs projets mais qui ne contribuent pas à améliorer la qualité de l'air et réduire l'extension urbaine; - au niveau social, le groupe a mené plusieurs actions citoyennes qui restent limitées dans le temps et ne touchent pas les vrais nécessiteux. Toutefois, le financement des activités des associations reste mitigé et non durable, des engagements non accomplis, comme les écoles qui ne sont pas raccordées en eau potable.

Mots clés: Développement durable, OCP, responsabilité sociale et environnementale

\section{Introduction}

Les pays industrialisés et ceux en voie de développement, les ONG, les médias ainsi que les différentes institutions publiques et privées ne sont plus indifférents aux risques encourus par notre planète si le rythme de développement actuel se poursuit sans prendre en considération ses conséquences (réchauffement de la planète, famine, sécheresse,..).

Le concept de développement durable a émergé dans les discours internationaux depuis le début des années 1990 et surtout lors de la conférence des nations unies sur l'environnement et le développement en 1992. Les résolutions de cette conférence ont mis l'accent sur l'urgence de changer les modes de production et de consommation pour s'orienter vers un développement permettant la préservation et le renouvellement des ressources naturelles.

Ce concept s'articule autour de trois éléments clés que sont : l'équité sociale, la préservation de l'environnement et l'efficacité économique.

Cet état de choses soulève la question de la volonté et de la faisabilité de la mise en place d'actions dans une dimension de développement durable de la part des chefs d'entreprises. Cependant la plupart des entreprises 
marocaines ne disposent pas des ressources nécessaires (ressources financières et humaines) pour faire face à leurs obligations notamment depuis l'adoption par le Maroc de réglementations visant à rendre les conséquences sociales et environnementales des groupes industriels plus transparentes.

Ce travail tente d'analyser les défis que doivent relever les entreprises marocaines notamment le groupe OCP (notre étude de cas) dans son site de Youssoufia pour se diriger vers le développement durable, examiner les stratégies permettant la mise en place d'une telle démarche et mettre l'accent sur les enjeux de l'adhésion à une telle vision.

Il a pour objet de répondre à la problématique suivante : quel est l'impact des projets initiés par l'OCP sur le développement de la ville de Youssoufia? Il se décline en trois parties: la première traite des soubassements théoriques du développement durable (DD), expose les défis que doivent relever les entreprises en matière de DD et les enjeux de l'adhésion à une telle démarche, la deuxième aborde la méthodologie poursuivie et la troisième est réservée aux résultats de l'étude exploratoire et à leurs discussions.

\section{Cadrage théorique}

\section{Définition du développement durable}

Le concept de développement durable (DD) est souvent défini comme un développement qui

« répond aux besoins du présent sans compromettre les capacités des générations futures à répondre aux leurs » (Brundtland, 1987). Appliqué par les entreprises, ce concept implique que la rentabilité demeure l'objectif principal de façon à assurer leur survie à long terme, mais que cette rentabilité ne peut être réalisée qu'à travers le respect de l'environnement, de la société et des hommes qui sont tout aussi importants et l'entreprise poursuivant une stratégie de DD doit parvenir à réaliser l'équilibre entre ces trois piliers (environnemental, social et économique). Pour Starik et Rands (1995), les activités durables se déclinent en cinq éléments. Outre les objectifs de prospérité économique, de justice sociale et de qualité environnementale, ils ajoutent la notion de compatibilité avec la culture de la société et la culture organisationnelle de façon à maximiser l'adoption de telles activités mais aussi à en assurer le succès.

\section{Les cadres théoriques traitant du DD}

Plusieurs théories économiques et organisationnelles ont essayé d'analyser les comportements des entreprises en matière de DD. Capron et Quairel-Lanoizelée (2004) opposent deux paradigmes. Le premier, 
regroupant les approches classiques et libérales (Friedman, 1971), la théorie des parties prenantes (Freeman, 1984) et la théorie de la dépendance envers les ressources (Pfeffer et Salancik, 1978), considère les relations comme " des contrats entre acteurs rationnels cherchant à maximiser des utilités convergentes et mettant en œuvre des stratégies aux impacts réels ». Le second paradigme regroupe les théories sociologiques néo-institutionnelles (Meyer et Rowan, 1977 ; Dimaggio et Powell, 1983 ; Oliver, 1991) et repose sur « une représentation symbolique des décisions de l'organisation, encastrée dans un réseau social et cherchant une légitimité, une image "convenable" malgré des attentes conflictuelles et contradictoires des acteurs du champ organisationnel ». L'approche par les ressources (Barney, 1991) a, quant à elle, été utilisée de façon plus instrumentale afin d'évaluer l'engagement des entreprises dans la voie du DD et de saisir les avantages à en tirer. Les approches combinant plusieurs perspectives et paradigmes existent également (Oliver, 1991, 1997), mais sont plus rares.

\section{Les défis du développement durable pour les entreprises}

Les sept points suivants définissent les plus grands défis auxquels sont confrontées les entreprises.

1. Développer une expertise en matière de développement durable : les contraintes de temps, de ressources humaines et financières et surtout le manque d'expertise réelle en matière de développement durable rend souvent complexes et difficiles le choix et la mise en œuvre d'actions et d'initiatives concrètes. Les entreprises expriment la nécessité de développer à l'interne ou d'avoir accès à une expertise qui leur permettra de gérer, de manière éclairée, les risques et les opportunités liés à une intégration du développement durable dans les operations et la stratégie de l'entreprise.

2. Valoriser le développement durable auprès des parties prenantes afin d'en faire un avantage compétitif : les bénéfices liés aux initiatives de développement durable en entreprise sont multiples (réduction des coûts de gestion des déchets, amélioration de l'image de marque de l'entreprise, meilleure réputation auprès des clients et des fournisseurs, ...).

Afin de rentabiliser les investissements liés au développement durable, les dirigeants des entreprises cherchent à valoriser leurs initiatives de développement durable auprès de leurs parties prenantes. Ainsi, devenir une « entreprise verte » constitue un choix stratégique de l'entreprise, qui devient concret par des initiatives et des actions pertinentes.

3. Informer les consommateurs pour améliorer leur réceptivité envers le développement durable: communiquer avec transparence est une manière de rentabiliser les investissements en matière de développement durable pour les entreprises; les consommateurs ne peuvent apprécier les initiatives de développement durable d'une compagnie s'ils ne les connaissent pas. Les 
entreprises doivent aussi démontrer la crédibilité des informations qu'elles avancent. D'autre part, le marché n'est pas toujours prêt à consommer de façon plus responsable.

Afin de mieux orienter leurs campagnes de publicité, les dirigeants des entreprises doivent choisir les informations permettant de mieux sensibiliser et d'impliquer les clients à consommer des produits et services durables (et souvent à payer plus cher pour ces produits et services).

4. Engager les parties prenantes afin qu'elles soutiennent l'entreprise dans sa démarche de développement durable: l'engagement des parties prenantes (internes et externes) est essentiel aux entreprises afin de soutenir et de valider leur démarche de développement durable. L'engagement des parties prenantes peut être défini comme une série d'activités menées par une entreprise dans le but d'établir des relations avec celle-ci. L'entreprise a généralement de nombreuses parties prenantes, tandis qu'elle est elle-même " partie prenante au sein de la communauté ${ }^{17}$ »). Les entreprises qui entretiennent une relation de confiance avec leurs parties prenantes bénéficient d'une meilleure acceptation de leurs produits et sont plus aptes à trouver des solutions communes ${ }^{18}$.

5. Conduire le changement à l'interne et ancrer le développement durable dans la culture organisationnelle de l'entreprise : entreprendre des initiatives de développement durable nécessite de mener des changements à l'interne et d'impliquer ses employés. Cependant, l'intégration du développement durable dans l'entreprise est souvent perçue comme une charge supplémentaire, complexe et floue aux yeux des employés et des fournisseurs. Ainsi, les dirigeants des entreprises sont confrontés parfois à des résistances de la part de certains employés.

L'intégration du développement durable à l'interne de l'entreprise consiste à faire en sorte qu'il soit perçu comme une extension logique de la mission de l'entreprise afin de façonner les pratiques internes et ancrer le développement durable à la culture organisationnelle.

6. Communiquer efficacement à l'interne afin d'attirer et de conserver des employés engagés : l'intégration du développement durable en entreprise nécessite le maintien d'une communication interne efficace et efficiente mais également des formations constantes auprès des employés. La formation offre aux employés la possibilité de mieux comprendre comment

${ }^{17}$ King, D. and Turcotte, M.-F. (2010), Introduction sur l'engagement des parties prenantes. Réseau entreprise et développement durable. Extrait de http://nbs.net/fr/connaissances/ engagement-des-parties prenantes /introduction/

${ }^{18}$ Heugens, P., Van den Bosch, F., \& Van Riel, C. (2002), Stakeholder integration: Building mutually enforcing relationships. Business \& Society, 41(1): 36-60. 
le développement durable est intégré concrètement aux opérations et activités de l'entreprise.

Cependant, une fois la formation effectuée, maintenir un engagement constant des employés en matière de développement durable constitue un défi. D’une part, les outils et pratiques de développement durable en entreprise sont en constante évolution (le cadre règlementaire, les nouvelles normes et certifications, ...). D'autre part, l'intégration du développement durable en entreprise nécessite l'utilisation de nouvelles méthodes de gestion dont l'efficacité n'est pas toujours vérifiée. Instaurer des voies de communication continue auprès des employés s'avère donc essentiel à l'atteinte des objectifs visés par l'entreprise. Toutefois, trop d'informations peuvent aussi diluer l'importance à accorder au développement durable. La recherche de l'équilibre est gage de succès.

7. Evoluer vers une intégration à long terme du développement durable dans la stratégie globale de l'entreprise: s'engager dans une démarche de développement durable à long terme passe souvent par une série d'initiatives et la réalisation de projets touchant à des aspects particuliers du développement durable. Plus ils sont porteurs, concrets, ambitieux, réalistes et permettent une progression par étapes, plus ils interpellent la direction et les employés et constituent une réussite.

\section{Les enjeux du développement durable pour les entreprises}

Stephany D (2003) fixe le cadre conceptuel du développement durable appliqué à l'entreprise en le définissant comme suit : «Le développement durable consiste pour une entreprise à assurer un développement, par une approche globale de la performance, maintenu dans le temps et résistant aux aléas, respectueux d'un système de valeurs explicité, impliquant différents acteurs internes et externes, dans une logique de progrès continu».

D’après cette définition, le développement dépasse le cadre économique pour intégrer des valeurs de progrès humains, comme l'a fait l'ONU en passant du PIB par habitant à l'Indice de Développement Humain qui intègre des dimensions relatives à la santé et à l'éducation des populations, et la mesure de la performance ne doit plus reposer uniquement sur des critères économiques, mais sur une approche globale.

Pour que le développement de l'entreprise devienne durable, il faut qu'elle intègre l'exigence du long terme dans tous ses compartiments. Egalement, l'entreprise doit prendre en considération les intérêts parfois contradictoires de différents acteurs qui sont susceptibles d'influencer ses décisions et sa façon de fonctionner.

Aujourd'hui, plusieurs facteurs d'ordre externe et interne obligent les entreprises à considérer les problématiques du développement durable. 
Il existe une relation d'interdépendance entre les entreprises et leurs parties prenantes qui sont constituées par les clients, les actionnaires, les salariés, les syndicats, les fournisseurs, les banques, les assurances, les médias, le gouvernement, le législateur, les associations et les ONG, les riverains, les

« activistes », les fonds communs de pension, les fonds de retraite, les fonds d'épargne, les universités, les clubs, etc. Ces parties prenantes influencent directement ou indirectement les choix des entreprises et influent sur leurs activités.

Pour identifier les enjeux du DD (crise, accident, émissions, nouvelle technologie, etc.), les entreprises consultent les parties prenantes et en contrepartie ces dernières formulent des attentes vis-vis des entreprises tels que la réduction des effets de leurs activités sur le patrimoine économique, social, sociétal et environnemental dont elles se sentent propriétaires, l'accès aux informations, la participation aux prises de décision. Mais cela peut être perçu comme une ingérence dans les choix des entreprises ce qui implique donc d'élaborer un arsenal juridique délimitant le droit de participation des parties prenantes pour éviter toute entrave au processus décisionnel des entreprises.

Le véritable enjeu du développement durable pour les entreprises est d'acquérir une légitimité pour entreprendre. Ainsi, elles doivent acquérir la confiance de leurs partenaires et prouver qu'elles se comportent bien. De plus la société civile fait pression pour évaluer et faire évoluer le comportement des entreprises envers leur environnement. Les entreprises récompensées sont celles qui agissent dans le sens du développement durable (légitimité, image positive,...) et les autres sont sanctionnées (pertes financières, baisse du capital,...).

L'enjeu externe ne constitue pas le seul motif pour être socialement et environnementalement responsable, des éléments internes propres à la dynamique de l'entreprise existent et sont décisifs pour sa compétitivité et sa pérennité.

Les enjeux internes qui incitent les entreprises à s'engager dans une démarche de développement durable passent d'abord par l'amélioration de leurs relations avec leurs partenaires sociaux.

- L'amélioration des relations avec les partenaires sociaux: les relations avec les principaux partenaires sociaux constituent un aspect parmi d'autres du développement durable et leur impact sur la pérennité des entreprises devient de plus en plus décisif.

- Les relations avec les clients : les clients sont devenus plus exigeants en demandant des produits de bonne qualité, des services sécurisés, des produits respectueux de l'environnement et en voulant être informés des 
conditions de production et de commercialisation des produits. Les entreprises engagées dans une démarche développement durable établissent une relation durable basée sur la confiance avec leurs clients ce qui leur permet de leur fidéliser et d'acquérir d'autres.

- Les relations avec les fournisseurs : les entreprises responsables exigent que leurs fournisseurs soient engagés dans une démarche de développement durable et élaborent avec eux une relation durable et de qualité ce qui permet de diminuer les coûts d'achats et de négocier les délais de paiement.

- Les relations avec la banque : les entreprises engagées dans une démarche de développement durable peuvent exiger de leurs banques qu'elles prennent en compte des critères non financiers tels que la gestion du risque environnemental et la gestion des droits de l'homme.

- Les relations avec les salariés : les employés dont les intérêts se croisent avec ceux de l'entreprise deviennent épanouis et mobilisés pour augmenter les profits en fournissant plus d'efforts et de meilleures productivités.

- Les relations avec les actionnaires : aujourd'hui, les actionnaires sont convaincus que la réalisation de bonnes performances économiques à long terme passe par la concrétisation de bonnes performances sociales et environnementales. Ainsi ils demandent à être associés dans la gestion de l'entreprise et mobiliser les salariés en leur permettant d'accéder à l'actionnariat.

- L'augmentation de la performance des entreprises : l'enjeu crucial de toute entreprise consiste à être en mesure de réaliser une performance supérieure à celle de ses concurrents. Bouquin (2004) représente la problématique générale de la performance comme suit :

Figure 1. La performance

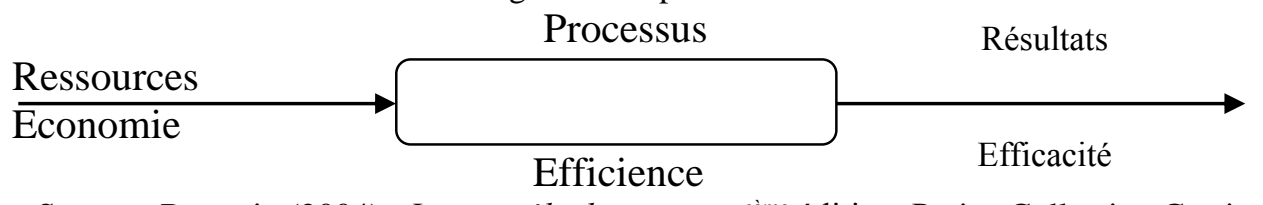

Source : Bouquin (2004), Le contrôle de gestion. $6^{\mathrm{ème}}$ édition, Paris : Collection Gestion.

Selon cet auteur, l'économie consiste à se procurer les ressources au moindre coût, l'efficience est le fait de maximiser la quantité obtenue de produits ou de services à partir d'une quantité donnée de ressources et l'efficacité est le fait d'atteindre les objectifs fixés.

L'accroissement de la performance se fait en intégrant le développement durable et combinant les trois éléments suivants que sont l'économie, l'efficience et l'efficacité. 
- L'économie : aujourd'hui, l'économie doit diminuer les impacts négatifs pour satisfaire les besoins de la société et optimiser les ressources qui doivent être utilisées au service du bien-être des générations présentes et futures. Pour réaliser des économies, les entreprises peuvent associer leurs parties prenantes pour diminuer les coûts liés aux litiges et de prévenir des dommages et se mettre en conformité avec les réglementations sociales et environnementales en vigueur.

- L'efficience : l'efficience s'obtient par l'entreprise qui encourage ses salariés à réaliser leur potentiel d'innovation dans le sens d'une qualité globale et incite ses managers à respecter les règles de sécurité, à améliorer la qualité des produits et de l'environnement.

- L'efficacité : les entreprises engagées dans une démarche de développement durable, réalisent une production responsable et accroissent leur efficacité grâce aux économies et aussi à l'innovation, ce qui leur permet d'optimiser les ressources et les compétences, de baisser les coûts, d'améliorer la productivité et augmenter l'efficacité.

- L'accroissement de la pérennité des entreprises : l'engagement des entreprises dans une démarche de développement durable leur permet d'élaborer une organisation plus performante et donc plus pérenne en modifiant leurs valeurs, leur culture, le comportement de leurs personnels et la façon de penser de leurs dirigeants. De plus ces entreprises socialement et environnementalement responsables, en préservant l'environnement, en réduisant les effets pervers de leurs activités, en sauvegardant l'état de santé de leurs salariés, participent efficacement à leur propre survie à long terme. Parce que la pollution, la santé et la sécurité des salariés et l'équilibre de la société sont des facteurs déterminants pour la survie des entreprises de ce siècle. Ne pas les intégrer, c'est mettre en péril la pérennité de l'entreprise.

\section{Approche méthodologique \\ Méthodologie poursuivie}

L'approche suivie repose sur l'élaboration de guides d'entretien semi directif, visant plusieurs intervenants et dont le but est d'étudier l'impact des projets tracés dans le cadre de la stratégie du développement durable que ce soit côté environnemental ou bien social répondant aux attentes des parties prenantes.

\section{Echantillonnage}

L'entretien ne prétend pas toucher l'ensemble des parties prenantes. Il s'agit d'un échantillon de la population composée des bénéficiaires ciblés, les responsables du groupe OCP site de Youssoufia, la commune urbaine, la société civile, les établissements d'enseignement, la province et la santé. 


\section{Résultats}

\section{Implantation de l'OCP à Youssoufia}

Depuis son installation à Youssoufia, le groupe d'OCP a cherché à ne pas trop éloigner les usines de traitement (lavage, séchage, calcination et traitement) des cités ouvrières, pour plusieurs raisons notamment le chargement et l'existence ou la proximité d'infrastructure. Toutefois, la localisation des sites de production obéit à des considérations purement géologiques.

Les installations techniques, implantées à l'intérieur des agglomérations, sont toujours opérationnelles et causent de nombreuses nuisances aux populations des quartiers voisins voire même la zone Ahmar. En effet, pendant plusieurs décennies, les habitants sont victimes de déversements quotidiens de dizaines de tonnes de poussières sur leurs habitations. Les vapeurs d'eau dégagées par les cheminées des usines sont fortement chargées de poussières.

Toutefois, l'OCP tente d'atténuer ces nuisances par des opérations dans le domaine d'environnement (reforestation), mais l'effort demeure insuffisant notamment du côté des quartiers non-OCP touchés. Ces installations minières intra-urbaines constituent également un sérieux obstacle pour l'extension de la ville. L'emprise foncière de ces unités limite l'urbanisation tout en l'orientant vers d'autres directions.

En outre des installations techniques, on trouve les terrils qui sont ces montagnes de résidus et d'impuretés extraites du minerai brut qui couvrent les zones de production. Les terrils ont un impact particulier sur le paysage des régions de production. Comme les usines de traitement, ils sont à l'origine des poussières qui sont transportées par les vents qui les déposent au voisinage, notamment sur les zones ${ }^{19}$ d'habitat. Ces terrils posent un véritable problème de gestion de l'environnement à l'OCP qui semble ne pas savoir quoi en faire.

Les carrières et les affaissements sont, en définitive, une conséquence inévitable des travaux miniers. Les affaissements sont fréquents dans la zone de Gantour où l'exploitation souterraine a toujours été importante. Ils peuvent avoir des conséquences graves sur les bâtiments, les canalisations et les moyens de communication. C'est une véritable destruction de la topographie naturelle alors qu'il s'agissait auparavant de terres agricoles.

Quand aux rejets liquides particulièrement polluants il faut noter que si les formes de dégradation de l'environnement générées par l'activité phosphatée sont connues, notamment par ceux qui les vivent

\footnotetext{
${ }^{19}$ Abdelaziz ADIDI : Les villes minières marocaines face au défi du développement durable, (source :

www.univ-lemans.fr/lettres/labo/gregum/activites/colloques/sfax/axe5/adidi.pdf)
} 
quotidiennement, en l'occurrence les mineurs et les habitants proches, il est admis aujourd'hui que l'OCP en tant que grand consommateur d'eau, notamment pour le lavage du phosphate, rejette des quantités liquides importantes composées de matériaux solides et de produits chimiques. De nombreuses communes, notamment celles abritant des usines de lavage, sont victimes de ces rejets en l'absence de digues permettant de contenir ces déversements. Il convient de signaler, par ailleurs, que la présence même du phosphate dans le sol et le sous-sol se traduit par des teneurs en fluorures des eaux des puits supérieures aux normes admissibles, ce qui détériore les dents des habitants et du bétail consommateurs de ces eaux et peuvent provoquer de graves maladies chez les nourrissons. Ces risques sanitaires s'ajoutent à ceux constitués par la présence de nitrates, également en teneurs excessives dans beaucoup de puits (engrais chimiques), dont certains sont en plus exposés à la pollution organique et microbienne découlant des rejets d'eaux usées des agglomérations amont (maladies hydriques). Il ressort donc de ce qui précède que l'espace minier phosphatier présente un paysage fortement altéré par une activité qui reste malgré ses retombées socio-économiques positives grande dévoreuse d'espace et destructrice de l'environnement.

Comme toute organisation, le site d'OCP à Youssoufia se doit désormais d'évaluer sa contribution, et d'endosser sa responsabilité face aux impacts directs ou indirects que le site engendre par ses activités et son fonctionnement. En 2011, le site a crée une entité développement durable pour pouvoir définir une politique de responsabilité sociétale, qui affirmera sa prise de conscience et sa volonté pour consolider son engagement envers la ville de Youssoufia et ses régions, et contribuer efficacement à leurs développement.

La fonction de responsabilité sociétale de l'entreprise qui est nouvellement créée dans l'organisation du site OCP de Youssoufia (début de l'année 2011), a pour ambition, notamment de contribuer à moyen et à long terme au développement des communautés qui l'entourent et de l'ensemble des acteurs de sa chaine de valeur. Le site veut être un acteur minier responsable et affirmer sa volonté d'être durablement intégré dans son environnement en contribuant au développement durable économique et sociétal local. L'OCP site de Youssoufia répond aux demandes de certaines parties prenantes soit par réponse affirmative ou négative, juste aux demandes stipulées par les parties prenantes actives et organisées. Cette opération de traitement des demandes doit se conformer à un certain nombre de critères:

- $\quad$ le territoire de l'action;

- l'impact de l'action;

- $\quad$ le porteur de l'action; 
- $\quad$ l'expérience du porteur de l'action;

- le domaine de l'action.

L'entreprise accorde une grande priorité aux demandes qui visent la lutte contre la pauvreté, la scolarisation, l'éducation ou le développement et l'embellissement de la ville, les demandes qui s'inscrivent dans le cadre de la santé, l'environnement, le sport ou les activités socioculturelles viennent en deuxième $\operatorname{rang}^{20}$.

\section{Résultats des entretiens et discussions}

Plusieurs projets ont été réalisés par l'OCP site de Youssoufia dans le coté social et environnemental au profit des riverains de cette ville. L'évaluation nous a permis de dégager les résultats suivants :

Au niveau environnemental l'entreprise a réalisé plusieurs projets : le projet de plantation des arbres, la construction d'une d'une station d'épuration des eaux usées (STEP) et le projet d'installation des filtres à manches. Ces projets visent l'amélioration de la qualité de l'air, la préservation des ressources hydriques et la réduction des émissions de gaz ainsi que la réduction d'extension urbaine.

D’après les entretiens avec les bénéficiaires on constate que:

- les espaces verts dans la ville Youssoufia et le nombre d'arbres plantés n'est pas suffisant, de plus, l'opération de plantation se concentre seulement dans la cité OCP; par conséquent, elle ne réduit pas l'extension urbaine et elle ne contribue pas dans l'amélioration de la qualité de l'air pollué de la ville ;

- l'entreprise prône maîtriser l'impact environnemental via l'installation des filtres à manches qui réduit 30 fois les émissions de gaz, le site contrôle quotidiennement ses émissions gazeux moyennent des analyseurs et en recourant aux prévisions météorologiques. Mais cette opération n'est pas suffisante vu les maladies respiratoires, dentaires...enregistrées chaque année, les terrains avoisinants sont devenus non cultivables, le phosphate trouvé chaque jour dans les rues et les toits des maisons. Donc l'entreprise devra assumer la responsabilité de ses émissions dans toute la ville et non pas seulement dans la limite de sa propriété et elle devra accélérer son opération de renouvellement de ses installations ;

- la STEP, est un moyen efficace pour l'optimisation de la demande en eau pour le lavage des phosphates, c'est un projet structurant qui va contribuer à la préservation des ressources naturelles et le recours aux ressources non conventionnelles. Il est à noter que le projet a connu du retard suite au conflit entre la commune et le site d'OCP à Youssoufia. Déjà plusieurs réclamations sont enregistrées par des associations suite à

${ }^{20}$ OCP site de Youssoufia 
l'exploitation excessive de la nappe d'eau que ce soit à Douar kacem, Loarrad, ... etc.

Au niveau social l'entreprise mène plusieurs actions citoyennes visant l'encouragement de la scolarisation en milieu rural et le renforcement d'un climat de solidarité dans la ville de Youssoufia, la création de plusieurs espaces sportifs et sociaux. Toutefois, l'étude terrain réalisée a fait ressortir plusieurs écarts:

- le financement des activités des associations est une action non durable car la responsabilité sociétale ne doit pas se limiter aux actions citoyennes ou en répondant aux demandes de sponsoring et de mécénat mais il faudrait qu'elle soit une initiative globale qui répond non seulement aux demandes des parties prenantes actives et organisées mais elle doit anticiper les intérêts de toutes les parties prenantes y compris celles qui ne stipulent pas leurs attentes à traves des demandes officielles, l'entreprise est obligée d'envisager la durabilité de ses actions. Par ailleurs, un leader mondial comme l'OCP doit mettre en place des outils permettant l'amélioration continue des processus de son fonctionnement;

- les locaux sanitaires sont crées, les bus scolaires sont distribués, les vélos et les fournitures scolaires sont distribués aussi, mais il faut raccorder les écoles en eau potable et en électricité, aussi les actions ne sont pas nombreuses, limitées dans le temps et dans l'espace et elles ne touchent pas les vrais nécessiteux, ainsi dans le secteur de la santé l'entreprise devra investir dans la santé des citoyens par la création d'un hôpital multidisciplinaire en assurant la gratuité des services médicaux;

- les projets initiés ne sont pas des grands projets qui vont faire bouger la ville, il faut y avoir un plan de développement réel et bien étudié afin de faire sortir la ville de son exclusion et donc une reconnaissance à Youssoufia surtout qu'elle est exploitée depuis 1931, outre sa position économique nationale, deuxième capitale mondiale des phosphates avec un gisement qui représente $35 \%$ des réserves reconnues à l'échelle nationale en tant que telle, elle devait refléter un développement social et économique harmonieux ;

-toutefois, les actions de sponsoring ou de mécénat ne représentent qu'une sorte de marketing social alors que la RSE est une démarche globale qui englobe ce qui est économique, social et environnemental.

- l'OCP site de Youssoufia a souhaité se doter d'une structure de management plaçant les communautés qui l'entourent et l'ensemble de ses acteurs au cœur des enjeux de l'entreprise. En ce sens, le concept de développement durable et la notion de la responsabilité sociétale répondent à ces attentes en proposant un système qui permet une amélioration continue de la qualité environnementale, sociale et économique fournie par 
l'entreprise. Ainsi le fait de s'engager dans une telle stratégie et pour que cette dernière soit légitime cela requiert une implication de tous les niveaux de responsabilité et une grande mobilisation de toute la plate forme ; cette façon de faire constitue une clé de succès de toute initiative ambitieuse.

- les points forts de la ville de Youssoufia sont la terre avec sa richesse en phosphates, l'eau souterraine non exploitée sauf par le groupe OCP, ainsi que les ressources humaines locales qui sont en majorité des jeunes. Ces jeunes ne sont pas impliqués dans la politique d'insertion ou de recrutement de l'entreprise. Donc les acteurs de Youssoufia doivent se baser sur ces piliers pour avoir un développement réel de la ville, les riverains devront se regrouper sous forme des associations pour défendre leurs droits.

Le défi de l'entreprise est le développement de la ville à travers l'enjeu de l'investissement, la communication et la participation de tous les acteurs. L'OCP doit s'ouvrir sur son entourage surtout la communauté locale en adoptant une approche participative lors de la prise de décision.

\section{Conclusion}

Le concept de développement durable a gagné le monde de l'entreprise et donné naissance à de nombreuses initiatives. Les grandes entreprises furent les premières à s'emparer de ce sujet, motivées par les exigences de la société civile et des consommateurs. Devant de telles pressions et face à l'engagement politique de faire du développement durable un nouveau paradigme, les entreprises se voient obligées de s'inscrire dans une démarche progressive de développement durable.

Certes les défis auxquels font face les entreprises qui s'engagent dans cette voie sont multiples et multidimensionnels (ressources financières et humaines limitées, manque de masse critique parmi les clients et les fournisseurs). Mais les entreprises responsables ont témoigné de nombreux impacts positifs tels que l'amélioration de leur image, l'augmentation de la loyauté de leurs salariés et leurs consommateurs et la réalisation des économies en matière de coûts d'exploitation. La collaboration avec les parties prenantes contribue à améliorer l'efficacité de la mise en place de stratégies de développement durable.

Pour notre étude de cas, nous pouvons affirmer que malgré les initiatives prises par le groupe OCP concernant le développement durable, il reste beaucoup à faire pour réduire les effets négatifs de ses activités pour la ville de Youssoufia.

\section{References:}

1. Barney, J. (1991), Firms resources and sustainable competitive advantage. Journal of Management, vol. 17, $\mathrm{n}^{\circ}$ 1: 99-120 
2. Bon Véronique. (2009), Le développement durable : des fondements pour l'entreprise aux écueils managériaux, Management \& Avenir, Vol. 9, $\mathrm{n}^{\circ} 29$ : 172-190.

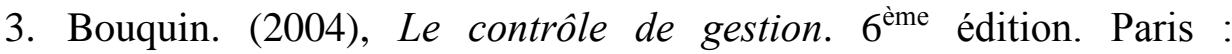
Collection Gestion.

4. Brundtland Gro Harlem. (1987), Notre avenir à tous, Commission mondiale sur l'environnement et le développement, Nations Unies.

5. Brunel S. (2007), Le développement durable, PUF, 127 pages.

6. Capron M et Quairel-Lanoizelée F. (2012), La responsabilité sociale d'entreprise, Edition La Découverte.

7. Dimaggio , P.J. et W.W. Powell. (1983), The iron cage revisited: institutional isomorphism and collective rationality in organizational fields, American Sociological Review, vol. 48, $\mathrm{n}^{\circ}$ 2: 147-160.

8. Donaldson T. (1999), Making Stakeholder Theory Whole, Academy of Management Review, Vol 24, ${ }^{\circ} 2$ : 237-241.

9. Dubigeon O. (2002), Mettre en pratique le développement durable, Quels processus pour l'entreprise responsable, Edition Village Mondial, Paris.

10. Freeman, R.E. (1984), Strategic Management: A Stakeholder Approach, Boston, Pitman.

11. Friedman M. (1971), Capitalisme et liberté, R. Laffont, Paris.

12. Gabriel P. (2004), Diffusion du développement durable dans le monde des affaires : un schéma conventionnel, Revue Française de Gestion, Vol 30, n¹52 : 199-214, septembre-octobre.

13. Gabriel P. et Cadiou C. (2005), Responsabilité sociale et environnementale et légitimité des entreprises : vers de nouveaux modes de gouvernance ?, La Revue des Sciences de Gestion, Direction et Gestion, $\mathrm{n}^{\circ} 211-212: 127-142$, janvier-avril.

14. Harrison J.S., Freman R.E. (1999), Stakeholders, Social Responsibility and Performance, Empirical Evidence and theoretical perspective. Academy of Management Review, Vol. 42.

15. Meyer, J. W., et Rowan, B. (1977), Institutionalized organizations: formal structure as myth and ceremony. Amercain Journal of Sociology, 83(2): 340-363.

16. Oliver, C. (1991), Strategic responses to institutional processes, Academy of Management Review, vol. 16, no 1: 145-179.

17. Oliver, C. (1997), Sustainable competitive advantage: combining institutional and resource-based views, Strategic Management Journal, vol. 18, $n^{\circ}$ 9: 697-713.

18. Pfeffer , J. et G.R. Salancik. 1978. The External Control of Organizations, New York, Harper and Row. 
19. PNUE. (2011), Vers une économie verte. Pour un développement durable et une éradication de la pauvreté. Synthèse à l'intention des décideurs.

20. Rapports annuels OCP, 2010, 2011 et 2012.

21. Rapport d'activités 2012, 2013 et 2014-Fondation OCP.

22. Rapport de la Direction de Développement Durable OCP-2013.

23. Starik , M. et G. Rands. (1995), Weaving an integrated Web: multilevel and multisystem perspective of ecologically sustainable organizations, The Academy of Management Review, vol. 20, $\mathrm{n}^{\circ} 4$ : 908-936.

24. Stephany D. (2003), Développement durable et performance de l'entreprise. Bâtir l'entreprise DD. Paris: Editions Liaisons. 